2014-09

\title{
Effect of Nonnutritive Sucking and Oral Stimulation on Feeding Performance in Preterm Infants
}

\section{Zhang, $Y$}

http://hdl.handle.net/10026.1/8283

10.1097/pcc.0000000000000182

Pediatric Critical Care Medicine

Ovid Technologies (Wolters Kluwer Health)

All content in PEARL is protected by copyright law. Author manuscripts are made available in accordance with publisher policies. Please cite only the published version using the details provided on the item record or document. In the absence of an open licence (e.g. Creative Commons), permissions for further reuse of content should be sought from the publisher or author. 
Effect of non-nutritive sucking and oral stimulation on feeding performance in preterm infants: A Randomized Controlled Trial

Yuxia Zhang, PhD, RN ${ }^{1}$; Tianchan Lv, MN, $\mathrm{RN}^{2}$; Xiaojing Hu, RN, $\mathrm{MN}^{2}$; Peng Shi, MSc ${ }^{3}$; Yun Cao, MD, $\mathrm{PhD}^{2}$; Jos M Latour, $\mathrm{PhD}, \mathrm{RN}^{4,5,6}$

${ }^{1}$ Nursing Department, Children's Hospital of Fudan University, Shanghai, P.R. China;

${ }^{2}$ Neonatal Intensive Care Unit, Children's Hospital of Fudan University, Shanghai, P.R. China;

${ }^{3}$ Department of Information, Children's Hospital of Fudan University, Shanghai, P.R. China;

${ }^{4}$ School of Nursing and Midwifery, Faculty of Health, Education and Society, Plymouth University, Plymouth, United Kingdom;

${ }^{5}$ School of Nursing and Midwifery, Faculty Health Science, Curtin University, Perth, Australia;

${ }^{6}$ Department of Pediatrics, Intensive Care Neonatology, Erasmus MC - Sophia Children's Hospital, Rotterdam, The Netherlands.

\section{Address for Correspondence:}

Yuxia Zhang, RN, PhD

Nursing Department, Children's Hospital of Fudan University, 399 Wan Yuan Road, Shanghai 201102, P.R. China

Tel/Fax: +8621 64931222

E-mail: yuxiazhang@aliyun.com

Copyright form disclosures: The authors have disclosed that they do not have any potential conflicts of interest. 


\section{ABSTRACT}

Objective: To evaluate the effectiveness of non-nutritive sucking (NNS) and oral stimulation (OS), either applied alone or in combination, to reduce the transition time from tube feeding to independent oral feeding.

Design: Randomized Controlled Trial.

Setting: A 40-bed neonatal intensive care unit in a university hospital in the P.R. of China.

Patients: A total of 120 preterm infants were admitted to the neonatal intensive care unit from December 2012 to July 2013.

Interventions: Oral motor interventions.

Measurements and Main Results: One-hundred and twelve preterm infants were assigned to three intervention groups (NNS, OS and combined NNS+OS) and one control group. Primary outcome was the number of days needed from introduction of oral feeding to autonomous oral feeding (transition time). Secondary outcomes measures were the rate of milk transfer ( $\mathrm{m} 1 \mathrm{~min}$ ), proficiency (intake first 5 minutes/volume ordered), volume transfer (volume transferred during entire feeding/volume prescribed), weight and hospital length of stay. Transition time was reduced in the three intervention groups compared to the control group $(p<0.001)$. The milk transfer rate in the three intervention groups was greater than in the control group $\left(F_{3,363}=15.37 ; \mathrm{p}<0.001\right)$. Proficiency in the NNS and OS groups did not exceed that in the control group while the NNS+OS group was greater than that in the control group at the stage when the infants initiated the oral feeding $(p=0.035)$. Among all groups, no significant difference was found on weight gain and length of stay.

Conclusions: The combined NNS+OS intervention reduced the transition time from introduction to independent oral feeding and enhanced the milk transfer rate. The combined intervention seems to have a beneficial effect on oral feeding proficiency in preterm infants. 
Key Words: Oral-motor; sensorimotor intervention; Sucking; Oral feeding; Feeding skill;

Prematurity 


\section{INTRODUCTION}

Medical technology advances have greatly increased survival rates of premature infants (1). Yet, most of them need to be admitted in a Neonatal Intensive Care Unit (NICU) because of a variety of medical and developmental complications (2). Oral feeding is one of the most common nursing care interventions in the care of newborn infants $(3,4)$. Moreover, oral feeding is a complex multisystem process involving the integration of lips, jaw, cheeks, tongue, palate, pharynx, and larynx (5). This is why preterm babies with immature neurological development and uncoordinated sucking-swallow-breath pattern cannot be fed by mouth successfully and safely (6). Competent suckle feeding, breast or bottle, is one of the hospital discharge criteria (7). In addition, Oral feeding problems have significant negative consequences for children's growth and development as well as their family's well-being (8, 9).

Oral motor intervention has been a topic of interest with clinical staffs working in NICU settings because of its direct targeted input to the oral structures involved in feeding. Currently there are a number of interventions to facilitate oral feeding in preterm infants (10-13). A systematic review explored the effect of oral motor interventions, focusing on nonnutritive sucking (NNS), oral/perioral stimulation (OS), and the combined intervention of NNS/OS (13). The review concluded that NNS alone showed increased sucking pressure during oral feeding and shortening the transition time from gavage feeding to total oral feeding. An earlier systematic review focusing on NNS only also confirmed preterm infants, receiving NNS, have better oral feeding performances and shorter length of hospital stay (14). However, NNS have been proven less effect on functional and oral feeding outcomes such as weight gain and growth in preterm infants (15). Evidence for the effectiveness of OS used alone is limited; two studies found that pre-feeding stimulation increased the milk 
intake in the first 5 minutes of nutritive sucking and improved weight gain $(16,17)$. Clearly, new studies are needed to further specify the effect of OS alone. Combination of NNS/OS reported positive changes in transition time, feeding performances and volume intake at oral feeding milestones (18-21). Furthermore, the combined intervention has positive effects on infants' intrinsic nutritive sucking skills (22). However, the effect of NNS plus OS on functional swallowing outcomes reported mix results such as negative effect on weight gain (13). To identify the underlying mechanism of these oral motor interventions, Fucile et al proposed NNS may have allowed the infants to engage oral musculature with greater endurance while OS may have strengthened these structures necessary for adequate sucking. The combination of NNS/OS may have enhanced suck-swallow-respiration coordination by providing a patterned input to the brain stem central pattern generator circuitry (18). To our knowledge, there are no studies available looking at the effectiveness of the combination of the intervention NNS and OS compared to the one of the single intervention.

The objective of our study was to compare and to evaluate the effectiveness of NNS and OS, either applied alone or in combination, to improve oral feeding in preterm infants.

\section{METHODS}

\section{Setting}

This study was conducted at the level 3 NICU of the Children's Hospital of Fudan University, Shanghai, P.R.China, a tertiary medical center. Infants admitted to the NICU are all outborn infants. The NICU is a 40-bed unit with a nurse to patient ratio of 1:2 depending on the patient acuity. Comprehensive care is provided to infants born at all gestational ages and birth weights with critical illness. Respiratory support includes conventional invasive mechanical 
ventilation, high-frequency ventilation and/or inhaled nitric oxide according to the severity of illness of the infants. Parental nutritional support is provided to infants who have not reached total enteral nutrition. The study was approved by the Ethics Committee of the Children's Hospital of Fudan University. Signed parental consent was obtained before participants' entry into the study.

\section{Study participants}

Power analysis showed that enrolment of 88 infants was required to detect a decrease of 4 days in the mean time from the introduction of nipple feeding to attainment of independent oral feeding, which has been established at $15 \pm 9$ days (23), with a type 1 error of 0.05 and a power of 0.80 . To allow for withdrawals, the actual number enrolled was $30 \%$ higher $(n=115)$.

One-hundred twenty were selected for the study, the eligibility criteria to participate in the trial were: 1) born between 29 to 34 weeks of gestational age (GA) as determined by obstetric ultra sonogram and clinical examination; 2) weight appropriate for gestational age; 3) Apgar scores of $\geq 3$ at 1 minute and $\geq 5$ at 5 minutes; 4) received all feedings by tube. Exclusion criteria were infants with congenital anomalies (oral, heart, et cetera) and infants who developed chronic medical complications during NICU admission, such as intraventricular hemorrhage grades III and IV, bronchopulmonary dysplasia, necrotizing enterocolitis. The excluded patients were not included in the final analysis. All included infants were born in other hospitals and transported within 24-48 hours to our NICU.

\section{Interventions}

After informed consent was provided by the parents, infants were randomized to one of four groups: NNS group, OS group, combined NNS and OS group, or the control group using a stratified blocked randomization. All interventions started 48 hours after discontinuation of 
nasal continuous positive airway pressure, and were continued until the newborn began exclusively oral diet. Interventions were not administered in case of proven apnea or bradycardia, or in case of a major disruption 30 minutes before the session (e.g. auditory examination). We monitored the patients from study onset to hospital discharge in the period December 2012 - July 2013.

Infants in the NNS group were allowed to suck on pacifiers for 5 min, 7-8 times a day, The pacifier was placed in the infant's mouth whether or not they have any attempt to suck, however, when necessary, the pacifier could be manipulated by the nurse to encourage sucking. OS was consisted of stroking the cheeks, lips, gums, and tongues which was according to the former $12 \mathrm{~min}$ oral stimulation of one program proposed by Fucile (18), infants in the OS group were stimulated once a day. The combined group was administered by the oral motor program including oral stimulation for $12 \mathrm{~min}$ and NNS for $3 \mathrm{~min}$, once a day. All infants in the three groups received the interventions $30 \mathrm{~min}$ before the beginning of scheduled feeding. Two experienced researchers (TL and $\mathrm{XH}$ ) were responsible for the administration of all interventions. Infants in the control group received the standard care. The standard care included: the infants received the early enteral nutrition and were fed every 2 hours, the nurses evaluated the gastrointestinal tolerance of infants by observing the color and quantity of gastric residual. Infants with birth weight between 1000-1500g were fed $1 \mathrm{ml}$ every time at first and the milk was added $2 \mathrm{ml} /$ day, while the infants with birth weight $>1500 \mathrm{~g}$ were fed $2 \mathrm{ml}$ every time at first and the milk was added $3-4 \mathrm{ml} /$ day. The initiation and advancement of oral feeding was left to the discretion of physician.

\section{Outcome measures}

Transition time was the primary outcome for this study, defined as the number of days needed from introduction of oral feeding to obtain autonomous oral feeding when the 
nasogastric tube was removed and all the daily milk volume was taken from the bottle. Infants' postmenstrual age (PMA) and weight at the two feeding milestones were recorded. The secondary outcomes were: 1) rate of transfer, i.e. the volume of milk consumed relative to the duration of the oral feeding session [m/min]; 2) proficiency, i.e. the volume of milk taken during the first 5 minutes as a percentage of the total [\%]; 3) volume transfer, i.e. the volume consumed as a percentage of the prescribed volume [\%]. The same recording protocol was applied for the four groups. The first observation was made on the day oral feeding was introduced (D1), the second observation was conducted three days later (D4). If the gavage was still necessary, a third recording was made another three days later (D7), and a final recording was made on the day when the infants reached autonomous feeding (DA). These parameters were monitored by a second researcher who was blind to the condition group allocation. Length of stay [days] was defined as the number of days from birth to discharge from the hospital. The average weight gain rate [\%] in hospitalized preterm infants was calculated by the method proposed by Patel et al. (24). We also recorded the illness degree using the NMI (Neonatal Medical Index) upon admission to the NICU. The NMI is a classification system used to review the medical course of preterm infants during hospitalization. NMI classifications fall in an ordinal scale from 1 to 5, with higher numbers denoting more serious medical complications. The behavioral state was measured at the start of the feeding session using the ABSS (Anderson Behavioral State Scale) (25). This scale determines the infant's behavioral state on a scale of 1 (deep sleep) to 12 (hard crying). Finally, episodes of apnea, bradycardia, and /or oxygen desaturation during the oral feeding session were also recorded.

\section{Statistical analyses}

Data are presented as means \pm SDs for continuous variables with normal distribution and 
percentages (\%) for categorical variables. One-way ANOVA and Fisher's exact test were used to compare infants baseline characteristics among the four groups. Because the influence of GA and birth weight on the outcomes, factorial design for ANCOVA analysis was used to access the effect of NNS, OS and the interaction of NNS and OS with the co-variables GA and birth weight. As duration of transition time differed in this study, we applied a mixed linear model to analyze the repeated measurements of rate of milk transfer, proficiency, and volume transfer with missing data. All statistical analyses were conducted using SAS v9.1.3 (SAS Institute Inc., Cary, NC, USA). A $p<0.05$ was regarded as statistically significant.

\section{RESULTS}

Of the 120 infants eligible for the study, 112 infants were randomized into the four groups and 108 infants concluded the study (Fig. 1).Age, gender, birth weight, Apgar score and severity of illness did not show significant differences among four group characteristics (Table 1). There was no difference in terms of behavioral state and numbers of episodes of apnea, bradycardia or oxygen desaturations.

The interaction of NNS and OS was not significant, so the interaction was not included in the final analysis models. The transition time for the control group was significantly longer than that of the NNS group $(p<0.001)$, the OS group $(p<0.001)$ and the combined group $(p<0.001)$, as shown in Table 2 . The combined group attained independent oral feeding at a significantly younger PMA $(p=0.004)$, lower weight $(p=0.01)$ and less days of life $(p=0.004)$ than those in the control group. PMA, weight and days of life did not significantly differ between the other two intervention groups and control group. There were no difference in PMA, weight and days of life among the three intervention groups (Table 2). 
ANCOVA computed on D1, D4, D7, DA revealed a significant effect for group $\left(F_{3,363}=15.37 ; p<0.001\right)$ and time $\left(F_{3,363}=68.12 ; p<0.001\right)$ on feeding transfer rate, whereas the group $\times$ time interaction was not significant $\left(F_{9,363}=1.43 ; p=0.172\right)$.Post-hoc group tests indicated that all three intervention group had significantly greater rate of transfer than the control group $(p<0.001)$ at D4, D7 and DA.There was no significant difference in transfer rate between any of the three intervention groups. Post-hoc time test indicated that the feeding transfer rate increased significantly from D1 to D4 $(p<0.001)$ and D7 to DA $(p<0.001)$, but not from D4 to D7 ( $p=0.412)$ (Fig.2).

There was a significant time effect for proficiency $\left(F_{3,363}=28.13 ; p<0.001\right)$, which increased from D1 to D4 $(p<0.001)$ and D7 to DA $(p=0.007)$, but not from D4 to D7 $(p=0.486)$. There was no significant group effect for proficiency $\left(F_{3,363}=1.74 ; p=0.158\right)$, however, the combined group had significantly greater proficiency than the control group at D1 ( $p=0.035)$ (Fig.3).Similarly, the volume transfer analysis indicated that there was a time effect for volume transfer $\left(F_{3,363}=25.91 ; p<0.001\right)$, which significantly increased from D1 to D4 $(p<0.001)$ and D7 to DA $(p=0.0056)$, but not from D4 to D7 $(p=0.327)$. There was no significant difference in volume transfer among the intervention groups and the control group $\left(F_{3,363}=1.72 ; p=0.162\right)$.

No significant difference occurred in length of hospital stay among the NNS group ( $38.0 \pm 13.9 \mathrm{~d})$, OS group $(40.4 \pm 13.9 \mathrm{~d})$, the combined group $(39.4 \pm 15.4 \mathrm{~d})$ and the control group $(41.4 \pm 12.9 \mathrm{~d})(\mathrm{p}=0.416)$. Also, no significant difference was found in weight gain rate during the hospital stay among the NNS group (10.4 $\pm 3.4 \%)$, OS group $(12.2 \pm 4.7 \%)$, the combined group $(11.3 \pm 4.0 \%)$ and the control group $(11.9 \pm 3.1 \%)(\mathrm{p}=0.466)$. 


\section{DISCUSSION}

Oral motor interventions are widely used to enhance the oral feeding of preterm infants.

However, faced with these approaches, nurses are challenged to determine which intervention is most effective. The results of our study confirm that preterm babies who received NNS, OS or combined oral motor interventions attained the autonomous oral feeding sooner than infants who did not receive such interventions. Compared to no intervention, NNS had a significantly positive effect on reducing the number of days to reach total oral feeding, which is consistent with the conclusions from a review (14). The moment of implementation and the duration of NNS, however, are widely ranging. In this study, NNS was administered for 5min prior to a tube feeding, 7-8 times a day. NNS provide sucking practice and the obvious sucking is to initiate the gastrointestinal cycle, thereby promoting earlier oral feeding(26). The current findings also suggest that pre-feeding OS alone can shorten the transition time when compared to the control group, in contrast with other studies $(16,17,27,28)$. This discrepancy may be owing to differences in the oral stimulation protocol while the duration of the stimulation protocol may influence the outcomes.

In relation to the reduction in transition time for the combined intervention group, our results are similar to the studies of Fucile et al. $(18,19)$, Rocha et al. $(21)$, and Fucile et al. (22), who reported a reduction of 7 days, 8.6 days and 10 days, respectively. Moreover, infants in our study were able to have shorter transition time when compared to other studies. These unexpected findings may be explained by the older GA inclusion criteria. At the same time, infants in the combined group were able to reach independent oral feeding at lower GA. The combination of the two interventions may lead to coordination of sucking-swallowing-breath and improved sucking skills (18) while the NNS and OS alone fails to induce an independent oral feeding at less mature GA stage. 
Compared to no intervention, the oral motor intervention has a beneficial effect on feeding performance. Our results indicate that the rate of milk transfer in the NNS group was significantly higher than in the control group, and several studies have corroborated this finding $(15,29)$. This outperformance may be related to better sucking pressure during feeding and more number of sucks per burst in the NNS group infants (30). In the present study, NNS had no beneficial effect on the infants' proficiency and volume taken. In contrast, Hill et al. (29) found that NNS increased the amount of formula consumed during the first 5 minutes of feeding. Hill proposed that NNS, provided before feeding, brought the infants to an optimal behavioral state for feeding, on account of which they could consume more formula during the first 5 minutes of feeding. Although the non-nutritive sucking is different from the nutritive sucking in terms of rate and rhythm (31), The fact that NNS can effectively modulate state behavior $(32,33)$, should be taken into account. However, when interpreting the results, this property may potentially confound the results of the intervention.

Oral stimulation improved the rate of milk transfer but not the proficiency and the volume taken when compared with no intervention, while Gaebler and Hanzlik's study indicated that pre-feeding oral stimulation had a small, not statistically significant effect on proficiency (17). This maybe owing to the rate of milk transfer reflect the overall oral motor ability while proficiency was used as an index of the actual oral feeding ability of infants (34),oral stimulation may contribute to the endurance rather than the oral motor skill.

We monitored the feeding performances during the transition time and the observation protocol was applied to trace the dynamic change. In the combined intervention group the rate of transfer at D4, D7 and DA was enhanced in comparison with the control infants. In addition, proficiency in the combined intervention group was higher than in the control group at D1, i.e. at the time when they start oral feeding, which is similar to findings from previous 
studies, in which sensory-motor-oral stimulation associated with NNS enhanced the feeding efficiency in the initial 5 min $(20,22)$, improved proficiency at the stage of one to two oral feedings a day (22). Moreover, the combined group had clinically greater proficiency at D1 than had the NNS group and the OS group, this finding may be helpful to distinguish the mechanisms underpinned these two oral motor interventions. NNS enhance satiation during tube feeding and to provide comfort (14), NNS elicit the sucking behavior of the preterm infants and this practice continue to develop the muscles needed for sucking and feeding. OS includes variation of stroking, tapping and stretching externally on the face or within the oral cavity. The centrally generated oral-motor patterns can be entrained to an applied stimulus, which serves to induce synchronous patterned neural activity. Therefore, from this perspective, NNS benefits the behavioral state and the internal gastrointestinal cycle while the stimuli of OS directly contribute to the development of suck and swallowing. Thus, this may be explained why this NNS/OS "patch" has addictive effect on the oral feeding performance for the preterm infants.

The combined intervention did not lead to an additive effect on infants' rate of transfer and volume transfer compared with either of the two oral motor interventions used alone. This may be explained by the shorter duration of NNS in the combined group, who received only 3 minutes NNS instead of the 5 minutes in the NNS groups, However, the combined group had better proficiency at the stage of initiating oral feeding and it could be possible that this group have outstanding oral feeding performances if the intervention prolonged the duration of NNS to some extent.

Although the three intervention groups reached full oral feeding earlier than the control group, length of stay did not significantly differ between the four groups, in contrast to findings from another study (21). There is no specific discharge plan in our hospital, this 
might have been a confounding variable in our study. At the same time, the oral motor intervention have no effect on the weight gain and growth of preterm infants in our study, which is similar to other studies $(19,21)$, the fact remains that infants weight gain and growth involves in many other factors.

The study has been performed in a level 3 NICU in a children's hospital without a perinatal department. This implicates that all infants are transferred from a women's hospital or other medical centers with an obstetric department. Although the majority of children's hospitals in the P.R. China are operated in a similar concept, in other regions of the world this might differ. The variety in nursing staffing levels might influence the nutrition practices. However, our major study finding can be considered within a range of available resources and admitted infants on the NICUs.

\section{LIMITATIONS}

Several limitations of our study warrant consideration. Firstly, the interventions were carried out by two researchers and it would be desirable to study the effect of the interventions performed by a large group of NICU nurses. Secondly, no parents were involved in the study because of the context of our NICU. Since parental participation is widely promoted in NICU services, future studies are needed to address parental involvement in feeding practices of their preterm infants. Thirdly, there was no follow-up when the infants were discharged from the hospital and this should be taken into consideration in a future study. Finally, the infants included in our study had no complex birth histories or treatment complications. The infants we excluded are highly at risk of feeding difficulties and special attention is needed to provide these infants optimal nutrition as this is directly associated with health outcomes. Additionally, all participating infants 
were out born and transported to our NICU. We were unable to measure differences in their early birth and transportation that may incur differences in baseline severity of pathology. The baseline characteristics of the infants were provide by the NMI. Indeed, this scale limits the presentation of influential factors of oral feeding such as invasive procedures or procedural medication and should be preformed in follow up studies.

\section{CONCLUSIONS}

In conclusion and in view of the benefits provided by oral motor interventions, the evidence of our study promotes the implementation of NNS and OS in NICU nursing care. Moreover, the combination of NNS/OS will have addictive effect on the oral feeding proficiency. The practicality of NNS and OS should be considered in the context of family-centered and developmental care. However, NICU nurses should also consider their knowledge and expertise along with the values and preference of the infants' parents when determining the best interventions. After all, for parents it may be easier to provide NNS rather than OS, while it may easier for nurses to implement OS.

\section{ACKNOWLEDGEMENT}

The authors thank the medical and nursing staff of the NICU at the Children's Hospital of Fudan University for their support and participation. We acknowledge Shaokang Zhan PhD and Huiting Tang MN who contributed to the study design and data analysis. We also thank Chantal Lau PhD for her critical review of the manuscript. 


\section{REFERENCES}

1. Blencowe H, Cousens S, Oestergaard MZ, et al: National, regional, and worldwide estimates of preterm birth rates in the year 2010 with time trends since 1990 for selected countries: a systematic analysis and implications. Lancet 2012;379: 2162-2172

2. Bick D: Born too soon: The global issue of preterm birth. Midwifery 2012; 28:401-402

3. Comrie JD, Helm JM: Common feeding problems in the intensive care nursery: Maturation, organization, evaluation, and management strategies. Semin Speech Lang $1997 ; 18: 239-261$

4. Jadcherla SR, Shaker R: Esophageal and upper esophageal sphincter motor function in babies. Am J Med 2001; 111 Suppl 8A:64S-68S

5. Delaney AL, Arvedson JC: Development of swallowing and feeding: Prenatal through first year of life. Dev Disabil Res Rev 2008; 14:105-117

6. Bulock F, Woolridge MW, Baum JD: Development of Coordination of Sucking, Swallowing and Breathing - Ultrasound Study of Term and Preterm Infants. Dev Med Child Neurol 1990; 32:669-678

7. Stark AR, Adamkin DH, Batton DG, et al: Hospital Discharge of the High-Risk Neonate Committee on Fetus and Newborn. Pediatrics 2008; 122:1119-1126

8. Dodrill P, McMahon S, Ward E, et al: Long-term oral sensitivity and feeding skills of low-risk pre-term infants. Early Hum Dev 2004; 76:23-37

9. Gisel EG: Eating problems and growth at 6 years of age in a whole population sample of extremely preterm children. Dev Med Child Neurol 2010; 52:230-230

10. Fucile S, Gisel E, Schanler RJ, et al: A Controlled-flow Vacuum-free Bottle System Enhances Preterm Infants' Nutritive Sucking Skills. Dysphagia 2009; 24:145-151 
11. White-Traut RC, Nelson MN, Silvestri JM, et al: Effect of auditory, tactile, visual, and vestibular intervention on length of stay, alertness, and feeding progression in preterm infants. Dev Med Child Neurol 2002; 44:91-97

12. Kumar A, Dabas P, Singh B: Spoon feeding results in early hospital discharge of low birth weight babies. J Perinatol 2010; 30:209-217

13. Arvedson J, Clark H, Lazarus C, et al: Evidence-Based Systematic Review: Effects of Oral Motor Interventions on Feeding and Swallowing in Preterm Infants. Am J Speech Lang Pathol 2010; 19:321-340

14. Pinelli J, Symington A: Non-nutritive sucking for promoting physiologic stability and nutrition in preterm infants. Cochrane Database Syst Rev 2005; CD001071

15. Sehgal SK, Prakash O, Gupta A, et al: Evaluation of beneficial effects of nonnutritive sucking in preterm infants. Indian Pediatr 1990; 27:263-266

16. Casesmith J: An Efficacy Study of Occupational-Therapy with High-Risk Neonates. Am J Occup Ther 1988; 42: 499-506

17. Gaebler CP, Hanzlik JR: The effects of a prefeeding stimulation program on preterm infants. Am J Occup Ther 1996; 50:184-192

18. Fucile S, Gisel E, Lau C: Oral stimulation accelerates the transition from tube to oral feeding in preterm infants. $J$ Pediatr 2002; 141:230-236

19. Fucile S, Gisel EG, Lau C: Effect of an oral stimulation program on sucking skill maturation of preterm infants. Dev Med Child Neurol 2005; 47:158-162

20. Hwang Y-S, Vergara E, Lin C-H, et al: Effects of Prefeeding Oral Stimulation on Feeding Performance of Preterm Infants. Indian J Pediatr 2010; 77:869-873

21. Rocha AD, Moreira MEL, Pimenta HP, et al: A randomized study of the efficacy of sensory-motor-oral stimulation and non-nutritive sucking in very low birthweight 
infant. Early Hum Dev 2007; 83:385-388

22. Fucile S, Gisel EG, McFarland DH, et al: Oral and non-oral sensorimotor interventions enhance oral feeding performance in preterm infants. Dev Med Child Neurol 2011; $53: 829-835$

23. Dodrill P, Donovan T, Cleghorn G, et al: Attainment of early feeding milestones in preterm neonates. J Perinatol 2008; 28:549-555

24. Patel AL, Engstrom JL, Meier PP, et al: Calculating postnatal growth velocity in very low birth weight (VLBW) premature infants. J Perinatol 2009; 29:618-622

25. Anderson GC, Behnke M, Gill NE, et al: Self-regulatory gavage-to-bottle feeding in preterm infants: Effects of behavioral state, energy expenditure, and weight gain. In. Key Aspects of Recovery: Nutrition, Rest, and Mobility. Funk SG, Tornquist EM, Champagne MT, Copp LA, Wiese RA. (Eds). New York: Springer, 1990, pp. 83-97.

26. Measel CP, Anderson GC: Nonnutritive sucking during tube feedings: effect on clinical course in premature infants. JOGN Nurs 1979; 8:265-72

27. Boiron M, Da Nobrega L, Roux S, et al: Effects of oral stimulation and oral support on nonnutritive sucking and feeding performance in preterm infants. Dev Med Child Neurol 2007; 49:439-444

28. Bragelien R, Rokke W, and Markestad T: Stimulation of sucking and swallowing to promote oral feeding in premature infants. Acta Paediatr 2007; 96:1430-1432

29. Hill AS: The Effects of Nonnutritive Sucking and Oral Support on the Feeding Efficiency of Preterm Infants. Newborn Inf Nurs Rev 2005; 5:133-141

30. Bernbaum JC, Pereira GR, Watkins JB, et al: Nonnutritive Sucking During Gavage Feeding Enhances Growth and Maturation in Premature-Infants. Pediatrics 1983; 71:41-45 
31. Harding C, Frank L, Dungu C, et al: The Use of Nonnutritive Sucking to Facilitate Oral Feeding in a Term Infant: A Single Case Study. J Pediatr Nurs 2012; 27:700-706

32. Woodson R, Drinkwin J, Hamilton C: Effects of Nonnutritive Sucking on State and Activity - Term-Preterm Comparisons. Infant Behav Dev 1985; 8:435-441

33. McCain GC: Behavioral state activity during nipple feedings for preterm infants. Neonatal Netw 1997; 16:43-47

34. Lau. C, Helene.R.Sheena., Shulman RJ, et al: Oral feeding in low birth weight infants. J Pediatr 1997; 130:561-569 


\section{Figure Legends}

Figure 1: Flow chart of RCT

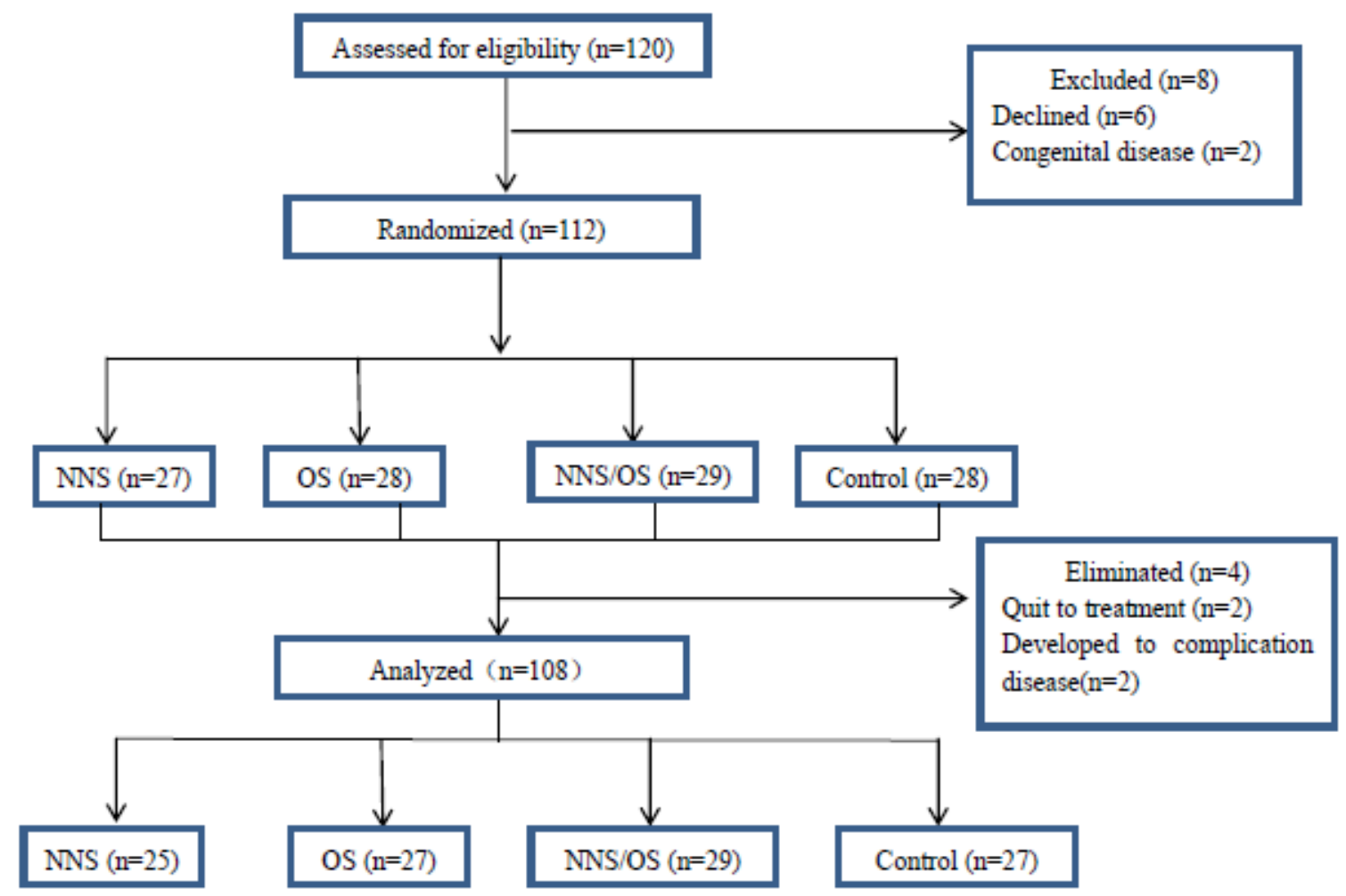

Figure 1: Flow chart of RCT

NNS, non-nutritive sucking; OS, oral stimulation. 
Table 1: Baseline characteristics of preterm infants in the four groups

Table 1: Baseline characteristics of preterm infants in the four groups

\begin{tabular}{lccccc}
\hline \multicolumn{1}{c}{ Characteristic } & NNS $(n=25)$ & OS $(n=27)$ & NNS+OS $(n=29)$ & Control $(n=27)$ & $p^{\mathrm{a}}$ \\
\hline Sex, $n(\%)$ & & & & & \\
\multicolumn{1}{c}{ Male } & $11(44.0)$ & $17(63.0)$ & $15(51.7)$ & $14(51.9)$ & $0.589^{b}$ \\
\multicolumn{1}{c}{ Female } & $14(56.0)$ & $10(37.0)$ & $14(48.3)$ & $13(48.1)$ & \\
Gestational age at birth (weeks) & $30.9 \pm 1.7$ & $31.1 \pm 1.3$ & $31.0 \pm 1.4$ & $31.1 \pm 1.2$ & 0.973 \\
Birthweight(g) & $1548.2 \pm 233.8$ & $1541.9 \pm 272.5$ & $1579.3 \pm 280.7$ & $1651.5 \pm 310.1$ & 0.452 \\
5 min Apgar score & $8.8 \pm 1.3$ & $8.5 \pm 1.5$ & $8.6 \pm 1.9$ & $8.6 \pm 1.7$ & 0.939 \\
Severity of illness (NMI) & $2.2 \pm 0.9$ & $2.4 \pm 0.9$ & $2.2 \pm 1.1$ & $2.3 \pm 0.9$ & 0.767 \\
\hline
\end{tabular}

${ }^{a}$ One-way ANOVA, ${ }^{b}$ Pearson Chi-Square test. NNS, non-nutritive sucking; OS, oral stimulation. NMI,Neonatal Medicine Index. 
Table 2: Number of days to transition from introduction to independent oral feeding

\begin{tabular}{lccccccc}
\multicolumn{1}{c}{} & Table 2 & \multicolumn{6}{c}{ Number of days to transition from introduction to independent oral feeding } \\
\hline \multirow{2}{*}{ Group } & \multicolumn{2}{c}{ Introduction of oral feeding } & \multicolumn{2}{c}{ Independent of oral feeding } & Transition time(days ) \\
\cline { 2 - 7 } & PMA & DOL & Weight & PMA & DOL & Weight & \\
\hline NNS $((n=25)$ & $33.4 \pm 1.3$ & $17.0 \pm 10.0$ & $1674.6 \pm 160.7$ & $34.8 \pm 1.3$ & $27.0 \pm 10.5$ & $1853.2 \pm 165.2$ & $10.0 \pm 5.0^{\mathrm{b}}$ \\
$\mathrm{OS}(n=27)$ & $33.7 \pm 1.1$ & $18.2 \pm 10.0$ & $1643.1 \pm 190.8$ & $35.1 \pm 1.2$ & $27.9 \pm 10.2$ & $1841.7 \pm 219.9$ & $9.6 \pm 4.8^{\mathrm{b}}$ \\
$\mathrm{NNS}+\mathrm{OS}(n=29)$ & $33.5 \pm 1.0$ & $17.7 \pm 10.1$ & $1665.0 \pm 171.3$ & $34.6 \pm 1.1^{\mathrm{b}}$ & $25.8 \pm 10.8^{\mathrm{b}}$ & $1838.1 \pm 161.8^{\mathrm{b}}$ & $8.1 \pm 2.7^{\mathrm{b}}$ \\
$\mathrm{Control}(n=27)$ & $33.3 \pm 1.1$ & $15.5 \pm 7.8$ & $1707.6 \pm 249.8$ & $35.4 \pm 1.4$ & $30.0 \pm 11.8$ & $1977.0 \pm 211.4$ & $14.6 \pm 6.8$ \\
$\mathrm{P}^{\mathrm{a}}$ & .718 & .725 & .912 & .017 & .018 & .059 & .000 \\
\hline
\end{tabular}

${ }^{a}$ ANCOVA post-hoc Tukey tests vs control. ${ }^{b}<0.05$. NNS, non-nutritive sucking; OS, oral stimulation. PMA, postmenstrual age (weeks); DOL, days of life. 


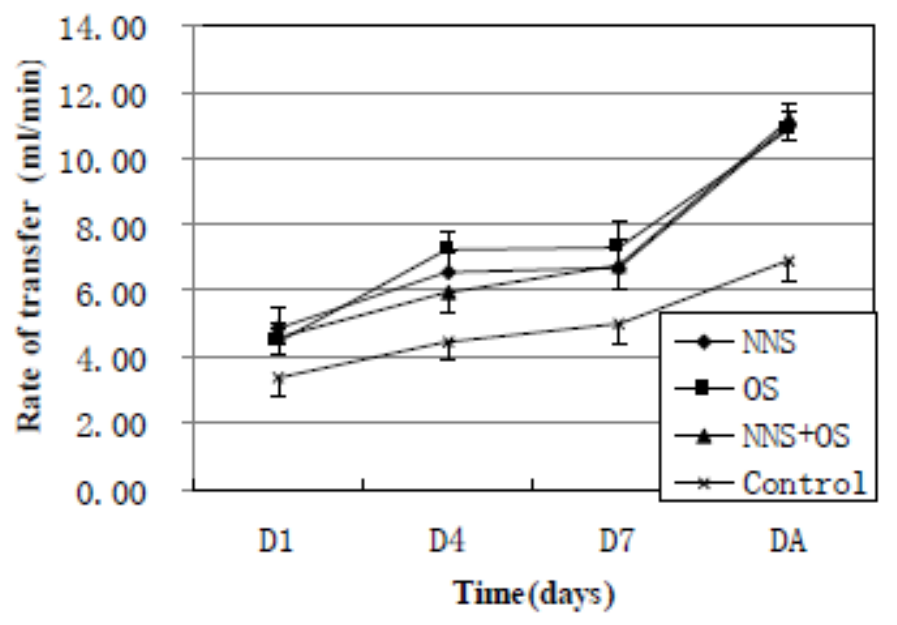

Figure 2: Rate of milk transfer according to time and group during the transition time for all three interventions groups and control group.

NNS, non-nutritive sucking; OS, oral stimulation. D1 represent the first observation and the second observation was conducted three days later (D4). If the gavage was still necessary, a third recording was made another three days later (D7), and a final recording was made on the day when the infants reached autonomous feeding (DA). 


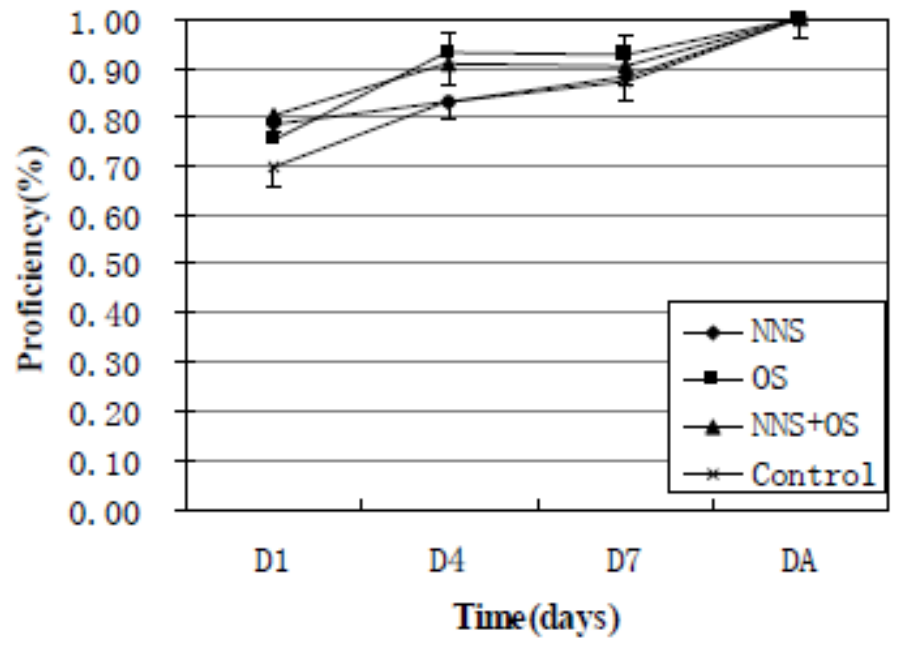

Figure 3: Proficiency according to time and group during the transition time for all three interventions groups and control group.

NNS, non-nutritive sucking; OS, oral stimulation. D1 represent the first observation and the second observation was conducted three days later (D4). If the gavage was still necessary, a third recording was made another three days later (D7), and a final recording was made on the day when the infants reached autonomous feeding (DA). 